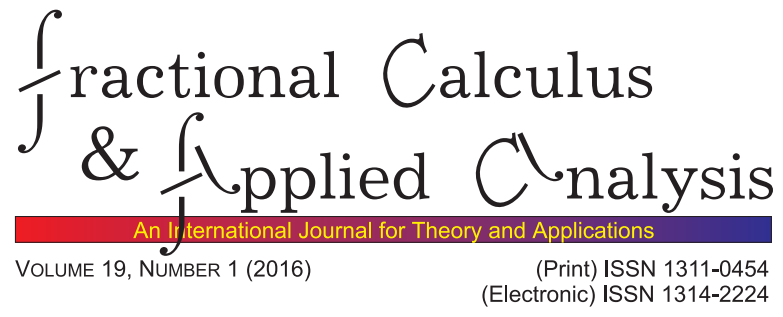

RESEARCH PAPER

\title{
EXISTENCE AND UNIQUENESS FOR A CLASS OF STOCHASTIC TIME FRACTIONAL SPACE PSEUDO-DIFFERENTIAL EQUATIONS
}

\author{
Ke Hu, Niels Jacob, Chenggui Yuan
}

\begin{abstract}
Some classes of stochastic fractional differential equations with respect to time and a pseudo-differential equation with respect to space are investigated. Using estimates for the Mittag-Leffler functions and a fixed point theorem, existence and uniqueness of mild solutions of the equations under consideration are established.
\end{abstract}

MSC 2010: Primary 34A08; Secondary 33E12, 35S05, 44A10, 60B15, 65C30

Key Words and Phrases: stochastic fractional differential equations, mild solutions, Brownian motion, Mittag-Leffler functions

\section{Introduction}

The theory of the fractional calculus has been developed for more than 300 years, we highlight the contributions of Leibniz, Liouville, Riemann and Weyl, just to name a few. There exists an extensive literature in this area, we here only mention Awawdeh, Rawashdeh and Jaradat [1], Gorenflo and Mainardi [3], Hilfer [5], Mainardi [13], Metzler and Klafter [15] and Podlubny [16] among others. Moreover, many mathematicians (see [11, [13] and [16]) have paid attention to the Riemann-Liouville fractional derivative and fractional differential equations composed by Riemann-Liouville fractional derivatives, i.e. equations of a form such as

$$
{ }_{0} \mathcal{D}_{t}^{\alpha} u(x, t)+\lambda u(x, t)=g(x, t), \text { for } 0<\alpha<1,
$$

(c) 2016 Diogenes Co., Sofia

pp. 56-68, DOI: 10.1515 /fca-2016-0004

DE GRUYTER 
for example under the initial data

$$
{ }_{0} \mathcal{D}_{t}^{\alpha-1} u(x, 0)=0 .
$$

The main tool for solving such equations is to use the Laplace transform.

On the other hand, it is well-known that many phenomena in various branches of science and industry can be modelled by stochastic differential equations (SDEs). Recently, attempts were made to combine fractional derivatives and SDEs. For example, El-Borai [2] has investigated the existence and uniqueness of stochastic fractional integro-differential equations. In this paper, motivated by the previous references, we will study the existence and uniqueness for a class of stochastic fractional differential equations. Our results are different from [2, since we use the Riemann-Liouville fractional derivatives.

In Section 2 we deal with auxiliary results, and in Section 3 we prove the existence and uniqueness for a class of stochastic fractional differential equations.

\section{Preliminaries}

Throughout this paper, let $(\Omega, \mathcal{F}, P)$ be a complete probability space with a filtration $\left\{\mathcal{F}_{t}\right\}_{t \geqslant 0}$ satisfying the usual conditions, i.e. it is right continuous and $\mathcal{F}_{0}$ contains all $P$-null sets. $B(t)$ is a standard Brownian motion defined on $(\Omega, \mathcal{F}, P)$. We consider a stochastic fractional differential equation of the form

${ }_{0} \mathcal{D}_{t}^{\alpha} u(x, t)+\psi\left(D_{x}\right) u(x, t)+p\left(x, D_{x}\right) u(x, t)-\int_{0}^{t} f(u(x, \theta)) d B(\theta)=g(x, t)$,

with the initial data

$$
\left.{ }_{0} \mathcal{D}_{t}^{\alpha-1} u(x, t)\right|_{t=0}=0,
$$

for $x \in \mathbb{R}, 0<t \leqslant T, T<\infty$. Here, $0<\alpha<1, \psi: \mathbb{R} \rightarrow \mathbb{R}$ is a continuous negative definite function of class $C^{2}$, satisfying the growth condition (2.11), and

$$
p\left(x, D_{x}\right) v(x)=(2 \pi)^{-1 / 2} \int_{\mathbb{R}} e^{i x \xi} p(x, \xi) \hat{v}(\xi) d \xi
$$

is a pseudo-differential operator with symbol $p: \mathbb{R} \times \mathbb{R} \rightarrow \mathbb{R}$. For the details on Itô's stochastic integral, we refer the reader to [12]. We will discuss later on certain properties which we have to impose on the symbol $p(x, \xi)$. In this paragraph, however, the only property needed is that $p\left(x, D_{x}\right)$ satisfies the estimate

$$
\left\|p\left(x, D_{x}\right) u(\cdot, t)\right\|_{L^{2}} \leqslant \mu\|u(\cdot, t)\|_{L^{2}}, \quad u(\cdot, t) \in L^{2}(\mathbb{R}),
$$

where $\mu$ is independent of $t$, and

$$
\|u(\cdot, t)\|_{L^{2}}^{2}=\int_{\mathbb{R}}|u(x, t)|^{2} d x .
$$


We refer to [7, Remark 3.2], where suitable conditions for (2.4) to hold were discussed.

Let $\omega \mapsto u(\omega, x, t)$ be a random variable, which we often write just as $u(x, t)$. The space $L^{2}\left(\Omega \times \mathbb{R} ; L^{\infty}([0, T])\right)$ defined by

$$
\begin{aligned}
& L^{2}\left(\Omega \times \mathbb{R} ; L^{\infty}([0, T])\right) \\
& :=\left\{u: \Omega \times \mathbb{R} \rightarrow L^{\infty}([0, T]) \mid\left(\mathbb{E} \int_{\mathbb{R}} \sup _{0 \leqslant t \leqslant T}|u(x, t)|^{2} d x\right)^{1 / 2}<\infty\right\}
\end{aligned}
$$

is a Banach space equipped with norm

$$
\|u\|_{L^{2}\left(\Omega \times \mathbb{R} ; L^{\infty}([0, T])\right)}=\left(\mathbb{E} \int_{\mathbb{R}} \sup _{0 \leqslant t \leqslant T}|u(x, t)|^{2} d x\right)^{1 / 2} .
$$

Comparing [16, Example 4.3, p.140], (2.1) can be rewritten as:

$$
\begin{aligned}
u(x, t) & \\
= & (2 \pi)^{-1 / 2} \int_{\mathbb{R}} \int_{0}^{t}(t-\tau)^{\alpha-1} g(x-y, \tau)\left(F^{-1}\left(E_{\alpha, \alpha}\left(-\psi(\cdot)(t-\tau)^{\alpha}\right)\right)\right)(y) d \tau d y \\
+ & (2 \pi)^{-1 / 2} \int_{\mathbb{R}} \int_{0}^{t}(t-\tau)^{\alpha-1}\left(\int_{0}^{\tau} f(u(x-y, \theta)) d B(\theta)\right) \\
& \times\left(F^{-1}\left(E_{\alpha, \alpha}\left(-\psi(\cdot)(t-\tau)^{\alpha}\right)\right)\right)(y) d \tau d y \\
+ & (2 \pi)^{-1 / 2} \int_{\mathbb{R}} \int_{0}^{t}(t-\tau)^{\alpha-1}\left(p\left(x, D_{x}\right) u(x-y, \tau)\right) \\
& \times\left(F^{-1}\left(E_{\alpha, \alpha}\left(-\psi(\cdot)(t-\tau)^{\alpha}\right)\right)\right)(y) d \tau d y,
\end{aligned}
$$

and the equality holds $P$-almost everywhere for which we will write $P$-a.e. Here by $E_{\alpha, \beta}(z)$ we denote the (2-indices) Mittag-Leffler function defined by

$$
E_{\alpha, \beta}(z):=\sum_{n=0}^{\infty} \frac{z^{n}}{\Gamma(\alpha n+\beta)} .
$$

This is an entire function and we refer to [11, [13] or [16] where basic properties of $E_{\alpha, \beta}(z)$ are discussed. We now estimate the Mittag-Leffler function $E_{\alpha, \alpha}\left(-\psi(\cdot)(t-\tau)^{\alpha}\right)$. For this we need the following theorem which can be found in [16, Section 1.2, p.16]. Let $\psi: \mathbb{R} \rightarrow \mathbb{R}$ be a continuous negative definite function. In fact we assume that $\psi$ has the representation

$$
\psi(\xi)=\int_{\mathbb{R} \backslash\{0\}}(1-\cos y \xi) \nu(d y)
$$

with a Lévy measure $\nu$ satisfying $\int_{\mathbb{R} \backslash\{0\}} 1 \wedge|y|^{2} \nu(d y)<\infty$. We assume further that $\psi$ is of class $C^{2}$, note that $\psi(\xi) \geqslant 0$, then for $l \leqslant 2$, 


$$
\left|\frac{d^{l} \psi(\xi)}{d \xi^{l}}\right| \leqslant \begin{cases}\psi(\xi), & l=0 \\ C_{1} \psi^{1 / 2}(\xi), & l=1 \\ C_{2}, & l=2 .\end{cases}
$$

Moreover, we require a lower bound for $\psi$, i.e.

$$
\psi(\xi) \geqslant C_{\psi}\left(1+|\xi|^{2}\right)^{\gamma / 2}
$$

for some $\gamma \in(1,2)$ and all $\xi \in \mathbb{R}$. We refer to [8]-[10] where examples of continuous negative definite functions are given. Using the table of Bernstein functions provided in [18, we can easily construct many examples of type $f\left(|\xi|^{2}\right)$ where $f$ is suitable Bernstein function.

\section{Existence and Uniqueness}

We start with a definition. A random function $u \in L^{2}\left(\Omega \times \mathbb{R} ; L^{\infty}([0, T])\right)$ is called a mild solution of the problem (2.1) and (2.2), if

$$
\begin{aligned}
& u(x, t) \\
& =(2 \pi)^{-1 / 2} \int_{\mathbb{R}} \int_{0}^{t}(t-\tau)^{\alpha-1} g(x-y, \tau)\left(F^{-1}\left(E_{\alpha, \alpha}\left(-\psi(\cdot)(t-\tau)^{\alpha}\right)\right)\right)(y) d \tau d y \\
& +(2 \pi)^{-1 / 2} \int_{\mathbb{R}} \int_{0}^{t}(t-\tau)^{\alpha-1}\left(\int_{0}^{\tau} f(u(x-y, \theta)) d B(\theta)\right) \\
& \quad \times\left(F^{-1}\left(E_{\alpha, \alpha}\left(-\psi(\cdot)(t-\tau)^{\alpha}\right)\right)\right)(y) d \tau d y \\
& +(2 \pi)^{-1 / 2} \int_{\mathbb{R}} \int_{0}^{t}(t-\tau)^{\alpha-1}\left(p\left(x, D_{x}\right) u(x-y, \tau)\right) \\
& \quad \times\left(F^{-1}\left(E_{\alpha, \alpha}\left(-\psi(\cdot)(t-\tau)^{\alpha}\right)\right)\right)(y) d \tau d y, \quad \text { P-a.e. }
\end{aligned}
$$

Let us introduce the operator

$$
\begin{aligned}
& L_{t} u(x, t) \\
& \begin{aligned}
= & (2 \pi)^{-1 / 2} \int_{\mathbb{R}} \int_{0}^{t}(t-\tau)^{\alpha-1} g(x-y, \tau)\left(F^{-1}\left(E_{\alpha, \alpha}\left(-\psi(\cdot)(t-\tau)^{\alpha}\right)\right)\right)(y) d \tau d y \\
+ & (2 \pi)^{-1 / 2} \int_{\mathbb{R}} \int_{0}^{t}(t-\tau)^{\alpha-1}\left(\int_{0}^{\tau} f(u(x-y, \theta)) d B(\theta)\right) \\
& \times\left(F^{-1}\left(E_{\alpha, \alpha}\left(-\psi(\cdot)(t-\tau)^{\alpha}\right)\right)\right)(y) d \tau d y \\
+ & (2 \pi)^{-1 / 2} \int_{\mathbb{R}} \int_{0}^{t}(t-\tau)^{\alpha-1}\left(p\left(x, D_{x}\right) u(x-y, \tau)\right) \\
& \times\left(F^{-1}\left(E_{\alpha, \alpha}\left(-\psi(\cdot)(t-\tau)^{\alpha}\right)\right)\right)(y) d \tau d y, \quad \text { P-a.e. }
\end{aligned}
\end{aligned}
$$


It follows that $u \in L^{2}\left(\Omega \times \mathbb{R} ; L^{\infty}([0, T])\right)$ is a mild solution to (2.1) and (2.2) if and only if $u$ is a fixed point of $L_{t}$, i.e.

$$
L_{t} u=u \text {. }
$$

TheOREM 3.1. Let $u, v \in L^{2}\left(\Omega \times \mathbb{R} ; L^{\infty}([0, T])\right)$ and let $f$ satisfy the Lipschitz conditions

$$
\|f(u)-f(v)\|_{L^{2}\left(\Omega \times \mathbb{R} ; L^{\infty}([0, T])\right)} \leqslant K_{1}\|u-v\|_{L^{2}\left(\Omega \times \mathbb{R} ; L^{\infty}([0, T])\right)},
$$

and the linear growth condition

$$
\|f(u)\|_{L^{2}\left(\Omega \times \mathbb{R} ; L^{\infty}([0, T])\right)} \leqslant K_{2}\left(1+\|u\|_{L^{2}\left(\Omega \times \mathbb{R} ; L^{\infty}([0, T])\right)}\right),
$$

where $K_{1}$ and $K_{2}$ are two positive constants. For $R>0$ consider

$$
B(g, R):=\left\{v \in L^{2}\left(\Omega \times \mathbb{R} ; L^{\infty}([0, T])\right) \mid\|v-g\|_{L^{2}\left(\Omega \times \mathbb{R} ; L^{\infty}([0, T])\right)} \leqslant R\right\}
$$

and assume

$$
\|g\|_{L^{2}\left(\Omega \times \mathbb{R} ; L^{\infty}([0, T])\right)} \leqslant \frac{R}{4} .
$$

Then there exists $T>0$ such that $L_{t}$ is a contraction on $L^{2}\left(\Omega \times \mathbb{R} ; L^{\infty}([0, T])\right)$ and $L_{t}$ leaves $B(g, R)$ invariant, i.e. $u \in B(g, R)$ implies $L_{t} u \in B(g, R)$. Consequently, $L_{t}$ has a unique fixed point which is a mild solution to the problem (2.1) and (2.2).

$$
\begin{aligned}
& \text { P r o o f. For } u, v \in L^{2}\left(\Omega \times \mathbb{R} ; L^{\infty}([0, T])\right) \text { we have } \\
& \left\|L_{t} u-L_{t} v\right\|_{L^{2}\left(\Omega \times \mathbb{R} ; L^{\infty}([0, T])\right)} \\
& =\|(2 \pi)^{-1 / 2} \int_{\mathbb{R}} \int_{0}^{t}(t-\tau)^{\alpha-1} \int_{0}^{\tau}(f(u(x-y, \theta))-f(v(x-y, \theta))) d B(\theta) \\
& \times\left(F^{-1}\left(E_{\alpha, \alpha}\left(-\psi(\cdot)(t-\tau)^{\alpha}\right)\right)\right)(y) d \tau d y \\
& +(2 \pi)^{-1 / 2} \int_{\mathbb{R}} \int_{0}^{t}(t-\tau)^{\alpha-1}\left(p\left(x, D_{x}\right)(u(x-y, \tau)-v(x-y, \tau))\right) \\
& \times\left(F^{-1}\left(E_{\alpha, \alpha}\left(-\psi(\cdot)(t-\tau)^{\alpha}\right)\right)\right)(y) d \tau d y \|_{L^{2}\left(\Omega \times \mathbb{R} ; L^{\infty}([0, T])\right)} \\
& \leqslant(2 \pi)^{-1 / 2} \| \int_{\mathbb{R}} \int_{0}^{t}(t-\tau)^{\alpha-1} \int_{0}^{\tau}(f(u(x-y, \theta))-f(v(x-y, \theta))) d B(\theta) \\
& \times\left(F^{-1}\left(E_{\alpha, \alpha}\left(-\psi(\cdot)(t-\tau)^{\alpha}\right)\right)\right)(y) d \tau d y \|_{L^{2}\left(\Omega \times \mathbb{R} ; L^{\infty}([0, T])\right)} \\
& +(2 \pi)^{-1 / 2} \| \int_{\mathbb{R}} \int_{0}^{t}(t-\tau)^{\alpha-1}\left(p\left(x, D_{x}\right)(u(x-y, \tau)-v(x-y, \tau))\right) \\
& \times\left(F^{-1}\left(E_{\alpha, \alpha}\left(-\psi(\cdot)(t-\tau)^{\alpha}\right)\right)\right)(y) d \tau d y \|_{L^{2}\left(\Omega \times \mathbb{R} ; L^{\infty}([0, T])\right)}
\end{aligned}
$$




$$
\begin{aligned}
& \leq(2 \pi)^{-1 / 2} \| \int_{0}^{t}(t-\tau)^{\alpha-1}\left(\int_{0}^{\tau}(f(u(\cdot, \theta))-f(v(\cdot, \theta))) d B(\theta)\right) \\
& *\left(F^{-1}\left(E_{\alpha, \alpha}\left(-\psi(\cdot)(t-\tau)^{\alpha}\right)\right)\right)(x) d \tau \|_{L^{2}\left(\Omega \times \mathbb{R} ; L^{\infty}([0, T])\right)} \\
&+(2 \pi)^{-1 / 2} \| \int_{0}^{t}(t-\tau)^{\alpha-1}\left(p\left(x, D_{x}\right)(u(\cdot, \tau)-v(\cdot, \tau))\right) \\
& {[4 p t] \quad *\left(F^{-1}\left(E_{\alpha, \alpha}\left(-\psi(\cdot)(t-\tau)^{\alpha}\right)\right)\right)(x) d \tau \|_{L^{2}\left(\Omega \times \mathbb{R} ; L^{\infty}([0, T])\right)} }
\end{aligned}
$$

where the $*$ means convolution. In (3.8), we estimate the first term

$$
\begin{aligned}
\| & \int_{0}^{t}(t-\tau)^{\alpha-1}\left(\int_{0}^{\tau}(f(u(\cdot, \theta))-f(v(\cdot, \theta))) d B(\theta)\right) \\
& *\left(F^{-1}\left(E_{\alpha, \alpha}\left(-\psi(\cdot)(t-\tau)^{\alpha}\right)\right)\right)(x) d \tau d y \|_{L^{2}\left(\Omega \times \mathbb{R} ; L^{\infty}([0, T])\right)} \\
{[4 p t]=} & \left\{\mathbb { E } \int _ { \mathbb { R } } \operatorname { s u p } _ { 0 \leqslant t \leqslant T } \left(\int_{0}^{t}(t-\tau)^{\alpha-1}\left(\int_{0}^{\tau}(f(u(\cdot, \theta))-f(v(\cdot, \theta))) d B(\theta)\right)\right.\right. \\
& \left.\left.*\left(F^{-1}\left(E_{\alpha, \alpha}\left(-\psi(\cdot)(t-\tau)^{\alpha}\right)\right)\right)(x) d \tau\right)^{2} d x\right\}^{1 / 2} \\
\leqslant & \left\{\mathbb { E } \int _ { \mathbb { R } } \left(\sup _{0 \leqslant t \leqslant T} \int_{0}^{t}(t-\tau)^{\alpha-1}\left(\int_{0}^{\tau}(f(u(\cdot, \theta))-f(v(\cdot, \theta))) d B(\theta)\right)\right.\right. \\
& \left.\left.*\left(F^{-1}\left(E_{\alpha, \alpha}\left(-\psi(\cdot)(t-\tau)^{\alpha}\right)\right)\right)(x) d \tau\right)^{2} d x\right\}^{1 / 2} \\
= & \left\{\mathbb { E } \left[\left(\int _ { \mathbb { R } } \left(\sup _{0 \leqslant t \leqslant T} \int_{0}^{t}(t-\tau)^{\alpha-1}\left(\int_{0}^{\tau}(f(u(\cdot, \theta))-f(v(\cdot, \theta))) d B(\theta)\right)\right.\right.\right.\right. \\
& \left.\left.\left.\left.*\left(F^{-1}\left(E_{\alpha, \alpha}\left(-\psi(\cdot)(t-\tau)^{\alpha}\right)\right)\right)(x) d \tau\right)^{2} d x\right)^{1 / 2}\right]^{2}\right\}^{1 / 2} \\
\leqslant & \left\{\mathbb { E } \left(\sup _{0 \leqslant t \leqslant T} \int_{0}^{t}(t-\tau)^{\alpha-1}\left\|\int_{0}^{\tau}(f(u(\cdot, \theta))-f(v(\cdot, \theta))) d B(\theta)\right\|_{L^{2}}\right.\right. \\
& \left.\left.\left.\times \| F^{-1}\left(E_{\alpha, \alpha}\left(-\psi(\cdot)(t-\tau)^{\alpha}\right)\right)\right)(x) d \tau \|_{L^{1}}\right)^{2}\right\}^{1 / 2}
\end{aligned}
$$




$$
\begin{aligned}
= & \left\{\mathbb { E } \left(\sup _{0 \leqslant t \leqslant T} \int_{0}^{t}(t-\tau)^{\alpha-1} h(t, \tau)\right.\right. \\
& \left.\left.\times\left\|\int_{0}^{\tau}(f(u(\cdot, \theta))-f(v(\cdot, \theta))) d B(\theta)\right\|_{L^{2}} d \tau\right)^{2}\right\}^{1 / 2},
\end{aligned}
$$

Here we have for $\alpha>0$

$$
h(t, \tau):=\left\|F^{-1}\left(E_{\alpha, \alpha}\left(-\psi(\cdot)(t-\tau)^{\alpha}\right)\right)\right\|_{L^{1}} .
$$

By Lemma 2.2 and Corollary 2.2 in [7], compare also with [6], we have already proven the integrability properties of $h(t, \tau)$ and $t \mapsto \tilde{h}(t, \tau):=$ $t^{\alpha-1} h(t, \tau)$ on every finite interval $[0, T]$. For $d>0$ we can find $T_{0}$ such that

$$
d \sup _{0 \leqslant t \leqslant T_{0}} \int_{0}^{t}(t-\tau)^{\alpha-1} h(t, \tau) d \tau \leqslant \kappa<1 .
$$

Now, by using Burkholder-Davis-Gundy inequality, compare [12], we arrive at

$$
\begin{aligned}
& \| \int_{0}^{t}(t-\tau)^{\alpha-1}\left(\int_{0}^{\tau}(f(u(\cdot, \theta))-f(v(\cdot, \theta))) d B(\theta)\right) \\
& \quad *\left(F^{-1}\left(E_{\alpha, \alpha}\left(-\psi(\cdot)(t-\tau)^{\alpha}\right)\right)\right)(x) d \tau d y \|_{L^{2}\left(\Omega \times \mathbb{R} ; L^{\infty}([0, T])\right)} \\
& \leqslant\left\{\mathbb { E } \left(\sup _{0 \leqslant t \leqslant T} \int_{0}^{t}(t-\tau)^{\alpha-1} h(t, \tau)\right.\right. \\
&\left.\left.\times\left\|\sup _{0 \leqslant \tau \leqslant T} \int_{0}^{\tau}(f(u(\cdot, \theta))-f(v(\cdot, \theta))) d B(\theta)\right\|_{L^{2}} d \tau\right)^{2}\right\}^{1 / 2} \\
& \leqslant\left(\frac{\kappa_{1}}{\alpha(\gamma-1)} T^{\alpha(\gamma-1) / \gamma}+\frac{\kappa_{2}}{\alpha} T^{\alpha}\right) \\
& \times\left\{\mathbb{E}\left(\left\|\sup _{0 \leqslant \tau \leqslant T} \int_{0}^{\tau}(f(u(\cdot, \theta))-f(v(\cdot, \theta))) d B(\theta)\right\|_{L^{2}}\right)^{2}\right\}^{1 / 2} \\
&=\left(\frac{\kappa_{1}}{\alpha(\gamma-1)} T^{\alpha(\gamma-1) / \gamma}+\frac{\kappa_{2}}{\alpha} T^{\alpha}\right) \\
& \times\left\{\mathbb{E} \int_{\mathbb{R} 0 \leqslant \tau \leqslant T} \sup _{0}\left(\int_{0}^{\tau}(f(u(\cdot, \theta))-f(v(\cdot, \theta))) d B(\theta)\right)^{2} d x\right\}^{1 / 2} \\
& \leqslant 4\left(\frac{\kappa_{1}}{\alpha(\gamma-1)} T^{\alpha(\gamma-1) / \gamma}+\frac{\kappa_{2}}{\alpha} T^{\alpha}\right) \\
& \times\left\{\int_{\mathbb{R}} \mathbb{E} \int_{0}^{\tau}(f(u(\cdot, \theta))-f(v(\cdot, \theta)))^{2} d \theta d x\right\}^{1 / 2}
\end{aligned}
$$




$$
\begin{aligned}
& \leqslant 4 T^{1 / 2}\left(\frac{\kappa_{1}}{\alpha(\gamma-1)} T^{\alpha(\gamma-1) / \gamma}+\frac{\kappa_{2}}{\alpha} T^{\alpha}\right) \\
& \times\left\{\mathbb{E} \int_{\mathbb{R}} \sup _{0 \leqslant t \leqslant T}(f(u(\cdot, \cdot))-f(v(\cdot, \cdot)))^{2} d x\right\}^{1 / 2} \\
& \leqslant 4 T^{1 / 2} K_{1}\left(\frac{\kappa_{1}}{\alpha(\gamma-1)} T^{\alpha(\gamma-1) / \gamma}+\frac{\kappa_{2}}{\alpha} T^{\alpha}\right)\|u-v\|_{L^{2}\left(\Omega \times \mathbb{R} ; L^{\infty}([0, T])\right)}
\end{aligned}
$$

For the second term in (3.8), we have

$$
\begin{aligned}
\| & \int_{0}^{t}(t-\tau)^{\alpha-1}\left(p\left(x, D_{x}\right)(u(\cdot, \tau)-v(\cdot, \tau))\right) \\
& *\left(F^{-1}\left(E_{\alpha, \alpha}\left(-\psi(\cdot)(t-\tau)^{\alpha}\right)\right)\right)(x) d \tau \|_{L^{2}\left(\Omega \times \mathbb{R} ; L^{\infty}([0, T])\right)} \\
= & \left\{\mathbb { E } \int _ { \mathbb { R } } \operatorname { s u p } _ { 0 \leqslant t \leqslant T } \left(\int_{0}^{t}(t-\tau)^{\alpha-1}\left(p\left(x, D_{x}\right)(u(\cdot, \tau)-v(\cdot, \tau))\right)\right.\right. \\
& \left.\left.*\left(F^{-1}\left(E_{\alpha, \alpha}\left(-\psi(\cdot)(t-\tau)^{\alpha}\right)\right)\right)(x) d \tau\right)^{2} d x\right\}^{1 / 2} \\
\leqslant & \left\{\mathbb { E } \int _ { \mathbb { R } } \left(\sup _{0 \leqslant t \leqslant T} \int_{0}^{t}(t-\tau)^{\alpha-1}\left(p\left(x, D_{x}\right)(u(\cdot, \tau)-v(\cdot, \tau))\right)\right.\right. \\
& \left.\left.*\left(F^{-1}\left(E_{\alpha, \alpha}\left(-\psi(\cdot)(t-\tau)^{\alpha}\right)\right)\right)(x) d \tau\right)^{2} d x\right\}^{1 / 2} \\
\leqslant & \left\{\mathbb{E}\left(\sup _{0 \leqslant t \leqslant T} \int_{0}^{t}(t-\tau)^{\alpha-1} h(t, \tau)\left\|p\left(x, D_{x}\right)(u(\cdot, \tau)-v(\cdot, \tau))\right\|_{L^{2}} d \tau\right)^{2}\right\}^{1 / 2},
\end{aligned}
$$

where

$$
h(t, \tau):=\left\|F^{-1}\left(E_{\alpha, \alpha}\left(-\psi(\cdot)(t-\tau)^{\alpha}\right)\right)\right\|_{L^{1}} .
$$

Using (2.4), we find

$$
\left\|p\left(x, D_{x}\right)(u(\cdot, \tau)-v(\cdot, \tau))\right\|_{L^{2}} \leqslant \mu\|u(\cdot, \tau)-v(\cdot, \tau)\|_{L^{2}}
$$

which implies that 


$$
\begin{aligned}
& \| \int_{0}^{t}(t-\tau)^{\alpha-1}\left(p\left(x, D_{x}\right)(u(\cdot, \tau)-v(\cdot, \tau))\right) \\
& *\left(F^{-1}\left(E_{\alpha, \alpha}\left(-\psi(\cdot)(t-\tau)^{\alpha}\right)\right)\right)(x) d \tau \|_{L^{2}\left(\Omega \times \mathbb{R} ; L^{\infty}([0, T])\right)} \\
& \leqslant\left\{\mathbb { E } \left(\sup _{0 \leqslant t \leqslant T} \int_{0}^{t}(t-\tau)^{\alpha-1} h(t, \tau)\right.\right. \\
& \left.\left.\times\left\|\sup _{0 \leqslant t \leqslant T} p\left(x, D_{x}\right)(u(\cdot, t)-v(\cdot, t))\right\|_{L^{2}} d \tau\right)^{2}\right\}^{1 / 2} \\
& \leqslant \mu\left(\frac{\kappa_{1}}{\alpha(\gamma-1)} T^{\alpha(\gamma-1) / \gamma}+\frac{\kappa_{2}}{\alpha} T^{\alpha}\right)\|u-v\|_{L^{2}\left(\Omega \times \mathbb{R} ; L^{\infty}([0, T])\right)} .
\end{aligned}
$$

Combining (3.10) and (3.12), we have

$$
\begin{aligned}
& \left\|L_{t} u-L_{t} v\right\|_{L^{2}\left(\Omega \times \mathbb{R} ; L^{\infty}([0, T])\right)} \leqslant(2 \pi)^{-1 / 2}\left(\frac{\kappa_{1}}{\alpha(\gamma-1)} T^{\alpha(\gamma-1) / \gamma}+\frac{\kappa_{2}}{\alpha} T^{\alpha}\right) \\
& \quad \times\left(4 K_{1} T^{1 / 2}+\mu\right)\|u-v\|_{L^{2}\left(\Omega \times \mathbb{R} ; L^{\infty}([0, T])\right)} .
\end{aligned}
$$

In (3.13), let $T$ be sufficiently small such that

$$
(2 \pi)^{-1 / 2}\left(\frac{\kappa_{1}}{\alpha(\gamma-1)} T^{\alpha(\gamma-1) / \gamma}+\frac{\kappa_{2}}{\alpha} T^{\alpha}\right)\left(4 K_{1} T^{1 / 2}+\mu\right)
$$

is less than or equal to 1 . Under this condition, $L_{t}$ is contractive.

Now, we note

$$
\begin{aligned}
& \left\|L_{t} u\right\|_{L^{2}\left(\Omega \times \mathbb{R} ; L^{\infty}([0, T])\right)} \\
& =\|(2 \pi)^{-1 / 2} \int_{\mathbb{R}} \int_{0}^{t}(t-\tau)^{\alpha-1} g(x-y, \tau) \\
& \quad \times\left(F^{-1}\left(E_{\alpha, \alpha}\left(-\psi(\cdot)(t-\tau)^{\alpha}\right)\right)\right)(y) d \tau d y \\
& +(2 \pi)^{-1 / 2} \int_{\mathbb{R}} \int_{0}^{t}(t-\tau)^{\alpha-1} \int_{0}^{\tau} f(u(x-y, \theta)) d B(\theta) \\
& \quad \times\left(F^{-1}\left(E_{\alpha, \alpha}\left(-\psi(\cdot)(t-\tau)^{\alpha}\right)\right)\right)(y) d \tau d y \\
& \left.+(2 \pi)^{-1 / 2} \int_{\mathbb{R}} \int_{0}^{t}(t-\tau)^{\alpha-1} p\left(x, D_{x}\right) u(x-y, \tau)\right) \\
& \quad \times\left(F^{-1}\left(E_{\alpha, \alpha}\left(-\psi(\cdot)(t-\tau)^{\alpha}\right)\right)\right)(y) d \tau d y \|_{L^{2}\left(\Omega \times \mathbb{R} ; L^{\infty}([0, T])\right)}
\end{aligned}
$$




$$
\begin{aligned}
\leqslant & (2 \pi)^{-1 / 2} \| \int_{\mathbb{R}} \int_{0}^{t}(t-\tau)^{\alpha-1} g(x-y, \tau) \\
& \times\left(F^{-1}\left(E_{\alpha, \alpha}\left(-\psi(\cdot)(t-\tau)^{\alpha}\right)\right)\right)(y) d \tau d y \|_{L^{2}\left(\Omega \times \mathbb{R} ; L^{\infty}([0, T])\right)} \\
+ & (2 \pi)^{-1 / 2} \| \int_{\mathbb{R}} \int_{0}^{t}(t-\tau)^{\alpha-1} \int_{0}^{\tau} f(u(x-y, \theta)) d B(\theta) \\
& \times\left(F^{-1}\left(E_{\alpha, \alpha}\left(-\psi(\cdot)(t-\tau)^{\alpha}\right)\right)\right)(y) d \tau d y \|_{L^{2}\left(\Omega \times \mathbb{R} ; L^{\infty}([0, T])\right)} \\
+ & (2 \pi)^{-1 / 2} \| \int_{\mathbb{R}} \int_{0}^{t}(t-\tau)^{\alpha-1} p\left(x, D_{x}\right) u(x-y, \tau) \\
& \times\left(F^{-1}\left(E_{\alpha, \alpha}\left(-\psi(\cdot)(t-\tau)^{\alpha}\right)\right)\right)(y) d \tau d y \|_{L^{2}\left(\Omega \times \mathbb{R} ; L^{\infty}([0, T])\right)}
\end{aligned}
$$

and further,

$$
\begin{aligned}
\| & L_{t} u \|_{L^{2}\left(\Omega \times \mathbb{R} ; L^{\infty}([0, T])\right)} \\
= & (2 \pi)^{-1 / 2} \| \int_{0}^{t}(t-\tau)^{\alpha-1} g(\cdot, \tau) \\
& *\left(F^{-1}\left(E_{\alpha, \alpha}\left(-\psi(\cdot)(t-\tau)^{\alpha}\right)\right)\right)(x) d \tau d y \|_{L^{2}\left(\Omega \times \mathbb{R} ; L^{\infty}([0, T])\right)} \\
& +(2 \pi)^{-1 / 2} \| \int_{0}^{t}(t-\tau)^{\alpha-1}\left(\int_{0}^{\tau} f(u(\cdot, \theta)) d B(\theta)\right) \\
& *\left(F^{-1}\left(E_{\alpha, \alpha}\left(-\psi(\cdot)(t-\tau)^{\alpha}\right)\right)\right)(x) d \tau \|_{L^{2}\left(\Omega \times \mathbb{R} ; L^{\infty}([0, T])\right)} \\
& +(2 \pi)^{-1 / 2} \| \int_{0}^{t}(t-\tau)^{\alpha-1} p\left(x, D_{x}\right) u(\cdot, \tau) \\
& *\left(F^{-1}\left(E_{\alpha, \alpha}\left(-\psi(\cdot)(t-\tau)^{\alpha}\right)\right)\right)(x) d \tau \|_{L^{2}\left(\Omega \times \mathbb{R} ; L^{\infty}([0, T])\right)} \\
\leqslant & 4(2 \pi)^{-1 / 2} T^{1 / 2}\left(\frac{\kappa_{1}}{\alpha(\gamma-1)} T^{\alpha(\gamma-1) / \gamma}+\frac{\kappa_{2}}{\alpha} T^{\alpha}\right)\|f(u(\cdot, t))\|_{L^{2}\left(\Omega \times \mathbb{R} ; L^{\infty}([0, T])\right)} \\
& +\mu(2 \pi)^{-1 / 2}\left(\frac{\kappa_{1}}{\alpha(\gamma-1)} T^{\alpha(\gamma-1) / \gamma}+\frac{\kappa_{2}}{\alpha} T^{\alpha}\right)\|u\|_{L^{2}\left(\Omega \times \mathbb{R} ; L^{\infty}([0, T])\right)} \\
& +(2 \pi)^{-1 / 2}\left(\frac{\kappa_{1}}{\alpha(\gamma-1)} T^{\alpha(\gamma-1) / \gamma}+\frac{\kappa_{2}}{\alpha} T^{\alpha}\right)\|g\|_{L^{2}\left(\Omega \times \mathbb{R} ; L^{\infty}([0, T])\right) \cdot}
\end{aligned}
$$

By using the linear growth condition (3.5), we have 


$$
\begin{aligned}
& \left\|L_{t} u\right\|_{L^{2}\left(\Omega \times \mathbb{R} ; L^{\infty}([0, T])\right)} \\
& \leqslant 4(2 \pi)^{-1 / 2} T^{1 / 2}\left(\frac{\kappa_{1}}{\alpha(\gamma-1)} T^{\alpha(\gamma-1) / \gamma}+\frac{\kappa_{2}}{\alpha} T^{\alpha}\right) K_{2}\left(1+\|u\|_{L^{2}\left(\Omega \times \mathbb{R} ; L^{\infty}([0, T])\right)}\right) \\
& \quad+\mu(2 \pi)^{-1 / 2}\left(\frac{\kappa_{1}}{\alpha(\gamma-1)} T^{\alpha(\gamma-1) / \gamma}+\frac{\kappa_{2}}{\alpha} T^{\alpha}\right)\|u\|_{L^{2}\left(\Omega \times \mathbb{R} ; L^{\infty}([0, T])\right)} \\
& \quad+(2 \pi)^{-1 / 2}\left(\frac{\kappa_{1}}{\alpha(\gamma-1)} T^{\alpha(\gamma-1) / \gamma}+\frac{\kappa_{2}}{\alpha} T^{\alpha}\right)\|g\|_{L^{2}\left(\Omega \times \mathbb{R} ; L^{\infty}([0, T])\right)} \\
& =C_{1}+C_{1}\|u\|_{L^{2}\left(\Omega \times \mathbb{R} ; L^{\infty}([0, T])\right)}+C_{2}\|u\|_{L^{2}\left(\Omega \times \mathbb{R} ; L^{\infty}([0, T])\right)} \\
& \quad+C_{3}\|g\|_{L^{2}\left(\Omega \times \mathbb{R} ; L^{\infty}([0, T])\right)},
\end{aligned}
$$

where

$$
\begin{array}{r}
C_{1}:=4(2 \pi)^{-1 / 2} T^{1 / 2}\left(\frac{\kappa_{1}}{\alpha(\gamma-1)} T^{\alpha(\gamma-1) / \gamma}+\frac{\kappa_{2}}{\alpha} T^{\alpha}\right) K_{2}, \\
C_{2}:=\mu(2 \pi)^{-1 / 2}\left(\frac{\kappa_{1}}{\alpha(\gamma-1)} T^{\alpha(\gamma-1) / \gamma}+\frac{\kappa_{2}}{\alpha} T^{\alpha}\right),
\end{array}
$$

and

$$
C_{3}:=(2 \pi)^{-1 / 2}\left(\frac{\kappa_{1}}{\alpha(\gamma-1)} T^{\alpha(\gamma-1) / \gamma}+\frac{\kappa_{2}}{\alpha} T^{\alpha}\right) .
$$

Thus we obtain

$$
\begin{aligned}
& \left\|L_{t} u-g\right\|_{L^{2}\left(\Omega \times \mathbb{R} ; L^{\infty}([0, T])\right)} \\
& \leqslant\left\|L_{t} u\right\|_{L^{2}\left(\Omega \times \mathbb{R}^{n} ; L^{\infty}([0, T])\right)}+\|g\|_{L^{2}\left(\Omega \times \mathbb{R} ; L^{\infty}([0, T])\right)} \\
& \leqslant C_{1}+C_{1}\|u\|_{L^{2}\left(\Omega \times \mathbb{R} ; L^{\infty}([0, T])\right)}+C_{2}\|u\|_{L^{2}\left(\Omega \times \mathbb{R} ; L^{\infty}([0, T])\right)} \\
& \quad+C_{3}\|g\|_{L^{2}\left(\Omega \times \mathbb{R} ; L^{\infty}([0, T])\right)}+\|g\|_{L^{2}\left(\Omega \times \mathbb{R} ; L^{\infty}([0, T])\right)} .
\end{aligned}
$$

In addition for $u \in B(g, R)$ it follows

$$
\|u\|_{L^{2}\left(\Omega \times \mathbb{R} ; L^{\infty}([0, T])\right)}-\|g\|_{L^{2}\left(\Omega \times \mathbb{R} ; L^{\infty}([0, T])\right)} \leqslant\|u-g\|_{L^{2}\left(\Omega \times \mathbb{R} ; L^{\infty}([0, T])\right)} \leqslant R,
$$

or

$$
\|u\|_{L^{2}\left(\Omega \times \mathbb{R} ; L^{\infty}([0, T])\right)} \leqslant \frac{5 R}{4} .
$$

Eventually, we arrive at

$$
\left\|L_{t} u-g\right\|_{L^{2}\left(\Omega \times \mathbb{R} ; L^{\infty}([0, T])\right)} \leqslant C_{1}+C_{1} \frac{5 R}{4}+C_{2} \frac{5 R}{4}+C_{3} \frac{R}{4}+\frac{R}{4} .
$$

We can make $C_{1}+C_{1} \frac{5 R}{4}+C_{2} \frac{5 R}{4}+C_{3} \frac{R}{4}+\frac{R}{4} \leqslant R$, i.e. $\frac{4 C_{1}}{3-5 C_{1}-5 C_{2}-C_{3}} \leqslant R$ and we find $\left\|L_{t} u-g\right\|_{L^{2}\left(\Omega \times \mathbb{R} ; L^{\infty}([0, T])\right)} \leqslant R$. 


\section{Acknowledgements}

The authors would like to thank the editors and the referees for detailed comments and valuable suggestions that helped to improve the paper significantly.

\section{References}

[1] A. Awawdeh, E.A. Rawashdeh, H.M. Jaradat, Analytic solution of fractional integro-differential equations. Annals of the University of Craiova, Mathematics and Computer Science Series 38 (2011), 1-10.

[2] M.M. El-Borai, On some stochastic fractional integro-differential equations. Adv. Dyn. Syst. Appl. 1 (2006), 49-57.

[3] R. Gorenflo, F. Mainardi, Random walk models for space-fractional diffusion processes. Fract. Calc. Appl. Anal. 1 (1998), 167-191.

[4] R. Gorenflo, A. Vivoli, F. Mainardi, Discrete and continuous random walk models for space-time fractional diffusion. Nonlinear Dyn. 38 (2004), 101-106.

[5] R. Hilfer, Threefold introduction to fractional derivatives. In: R. Klages, G. Radons, I.M. Sokolov (Eds.), Anomalous Transport: Foundations and Applications, Wiley-VCH, Weinheim (2008), 17-74.

[6] K. Hu, On an Equation Being a Fractional Differential Equation with Respect to Time and a Pseudo-differential Equation with Respect to Space Related to Lévy-Processes. Ph.D. Thesis, Swansea University, 2012.

[7] K. Hu, N. Jacob, C. Yuan, On an equation being a fractional differential equation processes. Fract. Calc. and Appl. Anal. 15 (2012), 128-140; DOI: $10.2478 / \mathrm{s} 13540-012-0009-0$; http://www.degruyter.com/view/j/fca.2012.15.issue-1/ issue-files/fca.2012.15.issue-1.xml.

[8] N. Jacob, Pseudo-differential Operators and Markov Processes, Vol. I: Fourier Analysis and Semigroups. Imperial College Press, London (2001).

[9] N. Jacob, Pseudo-differential Operators and Markov Processes, Vol. II: Generators and Their Potential Theory. Imperial College Press, London (2002).

[10] N. Jacob, Pseudo-differential Operators and Markov Processes, Vol. III: Markov Processes and Applications. Imperial College Press, London (2005).

[11] A.A. Kilbas, H.M. Srivastava, J.J. Trujillo, Theory and Applications of Fractional Differential Equations. North Holland Mathematics Studies 204, Elsevier, Amsterdam (2006). 
[12] X. Mao, Stochastic Differential Equations and Applications, 2nd Ed. Horwood Publishing Ltd. (2008).

[13] F. Mainardi, Fractional Calculus and Waves in Linear Viscoelasticity: An Introduction to Mathematical Models. Imperial College Press, London (2010).

[14] M.M. Meerschaert, E. Nane, P. Vellaisamy, Fractional Cauchy problems on bounded domains. Ann. Prob. 37 (2009), 979-1007.

[15] R. Metzler, J. Klafter, The Random walk's guide to anomalous diffusion: a fractional dynamical approach. Phys. Reports 339 (2000), 1-77.

[16] I. Podlubny, Fractional Differential Equations. Mathematics in Science and Engineering 198, Academic Press (1998).

[17] S.G. Samko, A.A. Kilbas, O.I. Marichev, Fractional Integrals and Derivatives: Theory and Applications. Gordon and Breach, Yverdon (1993).

[18] R.L. Schilling, R.M. Song, Z. Vondraček, Bernstein Functions: Theory and Applications. De Gruyter Studies in Mathematics, De Gruyter, Berlin (2010).

Swansea University

Department of Mathematics

Singleton Park

Swansea, SA2 8PP, U.K.

Received: April 12, 2015

e-mails: hukedr@126.com, N.Jacob@swansea.ac.uk,

C.Yuan@swansea.ac.uk

Please cite to this paper as published in:

Fract. Calc. Appl. Anal., Vol. 19, No 1 (2016), pp. 56-68, DOI: $10.1515 /$ fca-2016-0004 